\title{
Intersecting $k$-uniform families containing all the $k$-subsets of a given set
}

\author{
Wei-Tian $\mathrm{Li}^{*}$ \\ Department of Applied Mathematics \\ National Chung Hsing University \\ Taichung 40227, Taiwan \\ weitianli@dragon.nchu.edu.tw \\ Bor-Liang Chen \\ Department of Business Administration \\ National Taichung University of Science and Technology \\ Taichung 40401, Taiwan \\ blchen@ntit.edu.tw \\ Kuo-Ching Huang ${ }^{\dagger}$ \\ Department of Financial and Computational Mathematics \\ Providence University \\ Taichung 43301, Taiwan \\ kchuang@gm.pu.edu.tw \\ Ko-Wei Lih $\ddagger$ \\ Institute of Mathematics \\ Academia Sinica \\ Taipei 10617, Taiwan \\ makwlih@sinica.edu.tw
}

Submitted: Mar 18, 2013; Accepted: Aug 29, 2013; Published: Sep 13, 2013

Mathematics Subject Classifications: 05D05

\begin{abstract}
Let $m, n$, and $k$ be integers satisfying $0<k \leqslant n<2 k \leqslant m$. A family of sets $\mathcal{F}$ is called an $(m, n, k)$-intersecting family if $\left(\begin{array}{c}{[n]} \\ k\end{array}\right) \subseteq \mathcal{F} \subseteq\left(\begin{array}{c}{[m]} \\ k\end{array}\right)$ and any pair of members of $\mathcal{F}$ have nonempty intersection. Maximum $(m, k, k)$ - and $(m, k+1, k)$ intersecting families are determined by the theorems of Erdős-Ko-Rado and HiltonMilner, respectively. We determine the maximum families for the cases $n=2 k-1$, $2 k-2,2 k-3$, or $m$ sufficiently large.
\end{abstract}

Keywords: intersecting family; cross-intersecting family; Erdős-Ko-Rado; MilnerHilton; Kneser graph

\footnotetext{
${ }^{*}$ Research supported by NSC (No. 102-2115-M-005-001)

${ }^{\dagger}$ Research supported by NSC (No. 102-2115-M-126-002)

${ }^{\ddagger}$ Research supported by NSC (No. 102-2115-M-001-010)
} 


\section{Introduction}

For positive integers $a \leqslant b$, define $[a, b]=\{a, a+1, \ldots, b\}$ and let $[a]=[1, a]$. The cardinality of a set $X$ is denoted by $|X|$. A set of cardinality $n$ is called an $n$-set. A family of subsets of $X$ is said to be intersecting if no two members are disjoint. The family of all $k$-subsets of $X$ is denoted by $\left(\begin{array}{c}X \\ k\end{array}\right)$. Note that $\left(\begin{array}{c}{[m]} \\ k\end{array}\right)$ is intersecting if $0<k \leqslant m<2 k$. If all members of a family $\mathcal{F} \subseteq\left(\begin{array}{c}{[m]} \\ k\end{array}\right)$ contain a fixed element, then $\mathcal{F}$ is obviously an intersecting family and is said to be trivial. A trivial intersecting family can have at most $\left(\begin{array}{c}m-1 \\ k-1\end{array}\right)$ members. One of the cornerstones of the extremal theory of finite sets is the following pioneering result of Erdős, Ko, and Rado [5].

Theorem 1. Suppose $0<2 k<m$. Let $\mathcal{F} \subseteq\left(\begin{array}{c}{[m]} \\ k\end{array}\right)$ be an intersecting family. Then $|\mathcal{F}| \leqslant\left(\begin{array}{c}m-1 \\ k-1\end{array}\right)$. Moreover, the equality holds if and only if $\mathcal{F}$ consists of all $k$-subsets containing a fixed element.

Let $A \in\left(\begin{array}{c}{[m]} \\ k\end{array}\right)$ and $t \notin A$. Define $\mathcal{M}_{1}(A ; t)=\{A\} \cup\left\{B \in\left(\begin{array}{c}{[m]} \\ k\end{array}\right) \mid t \in B\right.$ and $\left.A \cap B \neq \emptyset\right\}$. Clearly $\left|\mathcal{M}_{1}(A ; t)\right|=\left(\begin{array}{c}m-1 \\ k-1\end{array}\right)-\left(\begin{array}{c}m-1-k \\ k-1\end{array}\right)+1$. Let $X \in\left(\begin{array}{c}{[m]} \\ 3\end{array}\right)$. Define $\mathcal{M}_{2}(X)=\left\{B \in\left(\begin{array}{c}{[m]} \\ k\end{array}\right) \mid\right.$ $|X \cap B| \geqslant 2\}$. Both $\mathcal{M}_{1}(A ; t)$ and $\mathcal{M}_{2}(X)$ are intersecting families. The largest size of a non-trivial intersecting family was determined in the following result of Hilton and Milner $[10]$.

Theorem 2. Suppose $0<2 k<m$. Let $\mathcal{F} \subseteq\left(\begin{array}{c}{[m]} \\ k\end{array}\right)$ be an intersecting family such that $\cap\{A \mid A \in \mathcal{F}\}=\emptyset$. Then $|\mathcal{F}| \leqslant\left(\begin{array}{c}m-1 \\ k-1\end{array}\right)-\left(\begin{array}{c}m-1-k \\ k-1\end{array}\right)+1$. Moreover, the equality holds if and only if $\mathcal{F}$ is of the form $\mathcal{M}_{1}(A ; t)$ or the form $\mathcal{M}_{2}(X)$, the latter occurs only for $k=3$.

In a more general form, the Erdö-Ko-Rado theorem describes the size and structure of the largest collection of $k$-subsets of an $n$-set having the property that the intersection of any two subsets contains at least $t$ elements. This theorem has motivated a great deal of development of finite extremal set theory since its first publication in 1961 . The complete establishment of the general form was achieved through cumulative works of Frankl [6], Wilson [12], and Ahlswede and Khachatrian [2]. Ahlswede and Khachatrian [1] even extended the Hilton-Milner theorem in the general case. The reader is referred to Deza and Frankl [4], Frankl [7], and Borg [3] for surveys on relevant results.

Let $0<k \leqslant n<2 k \leqslant m$. We call an intersecting family $\mathcal{F}$ an $(m, n, k)$-intersecting family if $\left(\begin{array}{c}{[n]} \\ k\end{array}\right) \subseteq \mathcal{F} \subseteq\left(\begin{array}{c}{[m]} \\ k\end{array}\right)$. Define $\alpha(m, n, k)=\max \{|\mathcal{F}| \mid \mathcal{F}$ is an $(m, n, k)$-intersecting family $\}$. An $(m, n, k)$-intersecting family with cardinality $\alpha(m, n, k)$ is called a maximum family. The focus for our study is the following.

Problem 3. For $0<k \leqslant n<2 k \leqslant m$, determine $\alpha(m, n, k)$ and the corresponding maximum families.

Suppose that $\mathcal{F}$ is an $(m, n, k)$-intersecting family. If any $A \in \mathcal{F}$ satisfies $|A \cap[n]| \leqslant$ $n-k$, then $|[n] \backslash A| \geqslant n-(n-k)=k$. Hence, there exists a $k$-subset $B \subseteq[n] \backslash A$. It is clear that $B \in \mathcal{F}$ and $B \cap A=\emptyset$, violating the intersecting condition on $\mathcal{F}$. Hence, we have a size constraint on any $A \in \mathcal{F}:|A \cap[n]| \geqslant n-k+1$, or equivalently, $|A \backslash[n]| \leqslant 2 k-n-1$. 
For any fixed $t \in[n]$, define $\mathcal{H}_{t}^{m, n, k}$ to be the family consisting of all $k$-subsets of $[n]$ and those $k$-subsets which contain $t$ and at least $n-k$ other elements from $[n]$, i.e.

$$
\mathcal{H}_{t}^{m, n, k}=\left(\begin{array}{c}
{[n]} \\
k
\end{array}\right) \cup \bigcup_{i=1}^{2 k-n-1}\left\{A \cup B \cup\{t\} \mid A \in\left(\begin{array}{c}
{[n] \backslash\{t\}} \\
k-i-1
\end{array}\right), B \in\left(\begin{array}{c}
{[n+1, m]} \\
i
\end{array}\right)\right\} .
$$

We often write $\mathcal{H}_{t}$ for $\mathcal{H}_{t}^{m, n, k}$ if the context is clear. It is easy to see that $\mathcal{H}_{t}$ is an $(m, n, k)$-intersecting family and its cardinality is equal to

$$
h(m, n, k)=\left(\begin{array}{l}
n \\
k
\end{array}\right)+\sum_{i=1}^{2 k-n-1}\left(\begin{array}{c}
n-1 \\
k-i-1
\end{array}\right)\left(\begin{array}{c}
m-n \\
i
\end{array}\right) .
$$

Hence, $\alpha(m, n, k) \geqslant h(m, n, k)$.

For the case $n=k$, Theorem 1 shows that $\alpha(m, k, k)=\left(\begin{array}{c}m-1 \\ k-1\end{array}\right)=h(m, n, k)$ and all maximum families are of the form $\mathcal{H}_{t}$ for some $t \in[k]$. For the case $n=k+1$, a maximum family is non-trivial since $\left(\begin{array}{c}{[k+1]} \\ k\end{array}\right)=\{[k+1] \backslash\{i\} \mid 1 \leqslant i \leqslant k+1\}$ and $\cap\left\{A \mid A \in\left(\begin{array}{c}{[k+1]} \\ k\end{array}\right)\right\}=\emptyset$. Theorem 2 shows that $\alpha(m, k+1, k)=\left(\begin{array}{c}m-1 \\ k-1\end{array}\right)-\left(\begin{array}{c}m-1-k \\ k-1\end{array}\right)+1=h(m, k+1, k)$ and all maximum families are of the form $\mathcal{M}_{1}(A ; t)=\mathcal{H}_{t}$, where $t \in[k+1]$ and $A=[k+1] \backslash\{t\}$, or the form $\mathcal{M}_{2}(X)$, where $X \in\left(\begin{array}{c}{[4]} \\ 3\end{array}\right)$, the latter occurs only for $k=3$.

In view of the above paragraph, the theorems of Erdös-Ko-Rado and Hilton-Milner can be regarded as special solutions to Problem 3. For these two particular cases, the obvious lower bound $h(m, n, k)$ coincides with the maximum value and, except the case for $k=3$ and $n=4$, all maximum families are of the form $\mathcal{H}_{t}$. This phenomenon leads us to pose the following.

Problem 4. When does $\alpha(m, n, k)=h(m, n, k)$ hold? When it does, are $\mathcal{H}_{t}$ 's the only maximum families?

In this paper, we give an affirmative answer $\alpha(m, n, k)=h(m, n, k)$ for the above questions when $n=2 k-1,2 k-2,2 k-3$, or $m$ sufficiently large.

\section{Main Tools}

Frequently, extremal problems concerning sub-families of $\left(\begin{array}{c}{[m]} \\ k\end{array}\right)$ can be translated into the context of Kneser graphs so that graph-theoretical tools may be employed to solve them. For $0<2 k \leqslant n$, a Kneser graph $\mathrm{KG}(n, k)$ has vertex set $\left(\begin{array}{c}{[n]} \\ k\end{array}\right)$ such that two vertices $A$ and $B$ are adjacent if and only if they are disjoint as subsets. By stipulation, we use $\mathrm{KG}(n, k)$ to denote the graph consisting of $\left(\begin{array}{l}n \\ k\end{array}\right)$ isolated vertices when $0<k \leqslant n<2 k$. An independent set in a graph is a set of vertices no two of which are adjacent. The maximum cardinality of an independent set in a graph $G$ is called the independence number of $G$ and is denoted by $\alpha(G)$. The Erdös-Ko-Rado theorem just gives the independence number of a Kneser graph and characterizes all maximum independent sets.

The direct product $G \times H$ of two graphs $G$ and $H$ is defined on the vertex set $\{(u, v) \mid$ $u \in G$ and $v \in H\}$ such that two vertices $\left(u_{1}, v_{1}\right)$ and $\left(u_{2}, v_{2}\right)$ are adjacent if and only if 
$u_{1}$ is adjacent to $u_{2}$ in $G$ and $v_{1}$ is adjacent to $v_{2}$ in $H$. The cardinality of the vertex set of a graph $G$ is denoted by $|G|$. The following result is due to Zhang [13].

Theorem 5. Let $G$ and $H$ be vertex-transitive graphs. Then $\alpha(G \times H)=\max \{\alpha(G)|H|$, $|G| \alpha(H)\}$. Furthermore, every maximum independent set of $G \times H$ is the pre-image of an independent set of $G$ or $H$ under projection.

Since Kneser graphs are vertex-transitive, we are going to use the above theorem for $G=\mathrm{KG}\left(n_{1}, k_{1}\right)$ and $H=\mathrm{KG}\left(n_{2}, k_{2}\right)$. The version of Theorem 5 for Kneser graphs was established in an earlier paper [8] of Frankl.

We can derive the following by Theorem 1, Theorem 5, and direct computation.

Lemma 6. When $2(k-i) \leqslant n$ and $2 i \leqslant m-n$,

$$
\alpha(\mathrm{KG}(n, k-i) \times \mathrm{KG}(m-n, i))= \begin{cases}\left(\begin{array}{c}
n-1 \\
k-i-1
\end{array}\right)\left(\begin{array}{c}
m-n \\
i
\end{array}\right) & \text { if } m \geqslant n k /(k-i), \\
\left(\begin{array}{c}
n \\
k-i
\end{array}\right)\left(\begin{array}{c}
m-n-1 \\
i-1
\end{array}\right) & \text { otherwise. }\end{cases}
$$

When $2(k-i)>n$ or $2 i>m-n, \alpha(\mathrm{KG}(n, k-i) \times \mathrm{KG}(m-n, i))=\left(\begin{array}{c}n \\ k-i\end{array}\right)\left(\begin{array}{c}m-n \\ i\end{array}\right)$.

Two families of sets $\mathcal{A}$ and $\mathcal{B}$ are said to be cross-intersecting if $A \cap B \neq \emptyset$ for any pair $A \in \mathcal{A}$ and $B \in \mathcal{B}$. Frankl and Tokushige [9] proved the following.

Theorem 7. Let $\mathcal{A} \subseteq\left(\begin{array}{c}X \\ a\end{array}\right)$ and $\mathcal{B} \subseteq\left(\begin{array}{c}X \\ b\end{array}\right)$ be nonempty cross-intersecting families of subsets of $X$. Suppose that $|X| \geqslant a+b$ and $a \leqslant b$. Then

$$
|\mathcal{A}|+|\mathcal{B}| \leqslant\left(\begin{array}{c}
|X| \\
b
\end{array}\right)-\left(\begin{array}{c}
|X|-a \\
b
\end{array}\right)+1
$$

The above inequality provides a useful tool for handling our problems.

\section{The cases for $m=2 k, n=2 k-1$, and $n=2 k-2$}

Proposition 8. We have $\alpha(2 k, n, k)=\frac{1}{2}\left(\begin{array}{c}2 k \\ k\end{array}\right)=h(2 k, n, k)$ for all $n(k \leqslant n<2 k)$.

This is true because any $(2 k, n, k)$-intersecting family cannot contain a $k$-subset and its complement in $[2 k]$ simultaneously. Any maximum family $\mathcal{F}$ can be obtained in the following manner. Pick a pair of a $k$-subset $A$ and its complement $A^{\prime}=[2 k] \backslash A$. If $A$ or $A^{\prime}$ is a subset of $[n]$, then we put it in $\mathcal{F}$. Otherwise, we put any one of them in $\mathcal{F}$.

A special case of the above construction for a maximum family is to choose the one that contains a prescribed element $t$ when neither $A$ nor $A^{\prime}$ is a subset of $[n]$. If $t \in[n]$, then the family so constructed is precisely $\mathcal{H}_{t}$.

Convention. From now on, we always assume that $0<k \leqslant n<2 k<m$ for any $(m, n, k)$-intersecting family. 
Proposition 9. For $n=2 k-1$ and all $m>2 k$, we have $\alpha(m, n, k)=\left(\begin{array}{l}n \\ k\end{array}\right)=h(m, n, k)$ and $\left(\begin{array}{c}{[n]} \\ k\end{array}\right)$ is the unique maximum $(m, n, k)$-intersecting family.

Proof. Let $\mathcal{F}$ be a maximum $(m, n, k)$-intersecting family. For any $A \in \mathcal{F}$, we know $k \geqslant|A \cap[n]| \geqslant n-k+1=k$. Thus, $A \in\left(\begin{array}{c}{[n]} \\ k\end{array}\right)$, and hence $\mathcal{F} \subseteq\left(\begin{array}{c}{[n]} \\ k\end{array}\right)$. Therefore, $\mathcal{F}=\left(\begin{array}{c}{[n]} \\ k\end{array}\right)$ and $\alpha(m, n, k)=|\mathcal{F}|=\left(\begin{array}{l}n \\ k\end{array}\right)=h(m, n, k)$. Note that all $\mathcal{H}_{t}$ 's are equal to $\left(\begin{array}{c}{[n]} \\ k\end{array}\right)$.

Suppose that $\mathcal{F}$ is an $(m, n, k)$-intersecting family. Define its canonical partition as follows.

$$
\mathcal{F}=\left(\begin{array}{c}
{[n]} \\
k
\end{array}\right) \cup\left(\bigcup_{i=1}^{2 k-n-1} \mathcal{F}_{i}\right)
$$

where $\mathcal{F}_{i}=\{F \in \mathcal{F}|| F \cap[n] \mid=k-i$ and $|F \cap[n+1, m]|=i\}$. For each $i$, we define an injection $f_{i}$ from $\mathcal{F}_{i}$ to the vertex set of $\mathrm{KG}(n, k-i) \times \mathrm{KG}(m-n, i)$ such that $f_{i}(F)=\left(A, B^{*}\right)$, where $A=F \cap[n]$ and $B^{*}=\{b-n \mid b \in F$ and $b \geqslant n+1\}$. Since $\mathcal{F}_{i}$ is intersecting, it is easy to verify that the image of $f_{i}$ is an independent set of $\mathrm{KG}(n, k-i) \times \mathrm{KG}(m-n, i)$. Thus, $\left|\mathcal{F}_{i}\right| \leqslant \alpha(\mathrm{KG}(n, k-i) \times \mathrm{KG}(m-n, i))$. We immediately obtain the following upper bound.

$$
|\mathcal{F}| \leqslant\left(\begin{array}{l}
n \\
k
\end{array}\right)+\sum_{i=1}^{2 k-n-1} \alpha(\mathrm{KG}(n, k-i) \times \mathrm{KG}(m-n, i)) .
$$

Theorem 10. For $n=2 k-2$, we have $\alpha(m, n, k)=h(m, n, k)$. All the maximum families are of the form $\left(\begin{array}{c}{[2 k-2]} \\ k\end{array}\right) \cup\left\{F \cup\{b\} \mid F \in \mathcal{F}^{*}, b \in[2 k-1, m]\right\}$, where $\mathcal{F}^{*}$ is any maximum intersecting family of $(k-1)$-subsets of $[2 k-2]$.

Proof. Let $\mathcal{F}$ be a largest $(m, 2 k-2, k)$-intersecting family with canonical partition $\left(\begin{array}{c}{[2 k-2]} \\ k\end{array}\right) \cup \mathcal{F}_{1}$. Now, all the conditions $2(k-1) \leqslant n, 2 \leqslant m-n$, and $m \geqslant n k /(k-1)$ hold. It follows from Lemma 6 that $\left|\mathcal{F}_{1}\right| \leqslant\left(\begin{array}{c}2 k-3 \\ k-2\end{array}\right)\left(\begin{array}{c}m-2 k+2 \\ 1\end{array}\right)$. Then $|\mathcal{F}|=\left(\begin{array}{c}2 k-2 \\ k\end{array}\right)+\left|\mathcal{F}_{1}\right| \leqslant$ $h(m, 2 k-2, k)$. As a consequence, $|\mathcal{F}|=h(m, 2 k-2, k)$ and $\left|\mathcal{F}_{1}\right|=\left(\begin{array}{c}2 k-3 \\ k-2\end{array}\right)\left(\begin{array}{c}m-2 k+2 \\ 1\end{array}\right)$. By Theorem $5, f_{1}\left(\mathcal{F}_{1}\right)$ is a maximum independent set in $\mathrm{KG}(2 k-2, k-1) \times \mathrm{KG}(m-2 k+2,1)$ and the collection $\mathcal{F}^{*}$ of all the first components of $f_{1}\left(\mathcal{F}_{1}\right)$ is an independent set of $\mathrm{KG}(2 k-2, k-1)$. Clearly, $\mathcal{F}^{*}$ is maximum because of its cardinality.

Remark. When $k=3$, an $(m, 2 k-2, k)$-family is also an $(m, k+1, k)$ family. There are other maximum families besides the collection of all $\mathcal{H}_{t}$ 's. This phenomenon is consistent with the Hilton-Milner theorem for the case $k=3$.

\section{The case for $n=2 k-3$}

Theorem 11. For $n=2 k-3$, we have $\alpha(m, n, k)=h(m, n, k)$. All the maximum families are of the form $\mathcal{H}_{t}$ for some $t \in[n]$. 
Proof. Let $\mathcal{F}$ be a largest $(m, 2 k-3, k)$-intersecting family with canonical partition $\left(\begin{array}{c}{[2 k-3]} \\ k\end{array}\right) \cup \mathcal{F}_{1} \cup \mathcal{F}_{2}$. We further partition $\mathcal{F}_{1}$ and $\mathcal{F}_{2}$ into subfamilies. Let $N=\left(\begin{array}{c}2 k-3 \\ k-1\end{array}\right)$. Partition $\left(\begin{array}{c}{[2 k-3]} \\ k-1\end{array}\right)$ into $A_{1}, \ldots, A_{N}$ and $\left(\begin{array}{c}{[2 k-3]} \\ k-2\end{array}\right)$ into $A_{1}^{\prime}, \ldots, A_{N}^{\prime}$ such that $A_{j} \cup A_{j}^{\prime}=[2 k-3]$ for all $j$. Define $\mathcal{F}\left(A_{j}\right)=\left\{F \in \mathcal{F} \mid F \cap[2 k-3]=A_{j}\right\}$ and $\mathcal{F}\left(A_{j}^{\prime}\right)=\left\{F \in \mathcal{F} \mid F \cap[2 k-3]=A_{j}^{\prime}\right\}$. Then

$$
\mathcal{F}=\left(\begin{array}{c}
{[2 k-3]} \\
k
\end{array}\right) \cup\left(\bigcup_{j=1}^{N}\left(\mathcal{F}\left(A_{j}\right) \cup \mathcal{F}\left(A_{j}^{\prime}\right)\right)\right)
$$

Observation. If $\mathcal{F}\left(A_{j}\right) \neq \emptyset$, then $\left|\mathcal{F}\left(A_{j}\right)\right|+\left|\mathcal{F}\left(A_{j}^{\prime}\right)\right| \leqslant m-2 k+3$.

If $\mathcal{F}\left(A_{j}^{\prime}\right)=\emptyset$, then $\left|\mathcal{F}\left(A_{j}\right)\right|+\left|\mathcal{F}\left(A_{j}^{\prime}\right)\right|=\left|\mathcal{F}\left(A_{j}\right)\right| \leqslant\left|\left\{A_{j} \cup\{b\} \mid b \in[2 k-2, m]\right\}\right|=$ $m-2 k+3$. If $\mathcal{F}\left(A_{j}^{\prime}\right) \neq \emptyset$, then $\left\{\{b\} \mid A_{j} \cup\{b\} \in \mathcal{F}\left(A_{j}\right)\right\} \subseteq\left(\begin{array}{c}{[2 k-2, m]} \\ 1\end{array}\right)$ and $\left\{\left\{b_{1}, b_{2}\right\} \mid A_{j}^{\prime} \cup\right.$ $\left.\left\{b_{1}, b_{2}\right\} \in \mathcal{F}\left(A_{j}^{\prime}\right)\right\} \subseteq\left(\begin{array}{c}{[2 k-2, m]} \\ 2\end{array}\right)$ are cross-intersecting. By Theorem $7,\left|\mathcal{F}\left(A_{j}\right)\right|+\left|\mathcal{F}\left(A_{j}^{\prime}\right)\right| \leqslant$ $\left(\begin{array}{c}m-2 k+3 \\ 2\end{array}\right)-\left(\begin{array}{c}m-2 k+2 \\ 2\end{array}\right)+1=m-2 k+3$. Hence, the observation holds.

Now suppose that all of $\mathcal{F}\left(A_{1}\right), \ldots, \mathcal{F}\left(A_{s}\right)$ are nonempty, yet $\mathcal{F}\left(A_{s+1}\right)=\cdots=$ $\mathcal{F}\left(A_{N}\right)=\emptyset$. Then we have

$$
|\mathcal{F}| \leqslant\left(\begin{array}{c}
2 k-3 \\
k
\end{array}\right)+s(m-2 k+3)+(N-s)\left(\begin{array}{c}
m-2 k+3 \\
2
\end{array}\right) .
$$

Case 1. $m \geqslant 2 k+2$.

Since $h(m, 2 k-3, k) \leqslant|\mathcal{F}|$ and $N=\left(\begin{array}{c}2 k-4 \\ k-2\end{array}\right)+\left(\begin{array}{c}2 k-4 \\ k-3\end{array}\right)$, it follows $s \leqslant\left(\begin{array}{c}2 k-4 \\ k-2\end{array}\right)$. We may assume $k \geqslant 5$ because $\alpha(m, 3,3)$ and $\alpha(m, 5,4)$ are known by the theorems of Erdös-KoRado and Hilton-Milner. It follows that $m \geqslant(2 k-3) k /(k-2)$. Together with $2(k-2)<$ $2 k-3$ and $4<m-2 k+3$, we have $\alpha(\mathrm{KG}(2 k-3, k-2) \times \mathrm{KG}(m-2 k+3,2))=\left(\begin{array}{c}2 k-4 \\ k-3\end{array}\right)\left(\begin{array}{c}m-2 k+3 \\ 2\end{array}\right)$ by Lemma 6 . Recall that $f_{2}\left(\mathcal{F}_{2}\right)$ is an independent set of $\mathrm{KG}(2 k-3, k-2) \times \mathrm{KG}(m-$ $2 k+3,2)$. Hence, $\left|\mathcal{F}_{2}\right| \leqslant\left(\begin{array}{c}2 k-4 \\ k-3\end{array}\right)\left(\begin{array}{c}m-2 k+3 \\ 2\end{array}\right)$. If $s<\left(\begin{array}{c}2 k-4 \\ k-2\end{array}\right)$, then $\left|\mathcal{F}_{1}\right|=\sum_{j=1}^{s}\left|\mathcal{F}\left(A_{j}\right)\right|<$ $\left(\begin{array}{c}2 k-4 \\ k-2\end{array}\right)(m-2 k+3)$. This leads to $|\mathcal{F}|=\left(\begin{array}{c}2 k-3 \\ k\end{array}\right)+\left|\mathcal{F}_{1}\right|+\left|\mathcal{F}_{2}\right|<h(m, 2 k-3, k)$, a contradiction. Thus, $s=\left(\begin{array}{c}2 k-4 \\ k-2\end{array}\right)$ and $\alpha(m, 2 k-3, k)=h(m, 2 k-3, k)$ for $m \geqslant 2 k+2$.

Case 2. $m=2 k+1$.

Suppose that $\left(\begin{array}{c}2 k-3 \\ k\end{array}\right)+\left(\begin{array}{c}2 k-4 \\ k-2\end{array}\right)\left(\begin{array}{l}4 \\ 1\end{array}\right)+\left(\begin{array}{c}2 k-4 \\ k-3\end{array}\right)\left(\begin{array}{l}4 \\ 2\end{array}\right)=h(2 k+1,2 k-3, k)<|\mathcal{F}|$. Since $N=\left(\begin{array}{c}2 k-4 \\ k-2\end{array}\right)+\left(\begin{array}{c}2 k-4 \\ k-3\end{array}\right)$, it follows from inequality (1) that $\left|\left\{j|| \mathcal{F}\left(A_{j}\right)|+| \mathcal{F}\left(A_{j}^{\prime}\right) \mid \geqslant 5\right\}\right|>$ $\left(\begin{array}{c}2 k-4 \\ k-3\end{array}\right)$. By our Observation, $\left|\mathcal{F}\left(A_{j}\right)\right|+\left|\mathcal{F}\left(A_{j}^{\prime}\right)\right| \geqslant 5$ implies $\mathcal{F}\left(A_{j}\right)=\emptyset$ for any $j$. Thus $\left|\left\{A_{j}^{\prime}|| \mathcal{F}\left(A_{j}^{\prime}\right) \mid \geqslant 5\right\}\right|>\left(\begin{array}{c}2 k-4 \\ k-3\end{array}\right)$. By Theorem 1 , there exist disjoint sets $A_{j_{1}}^{\prime}$ and $A_{j_{2}}^{\prime}$ in $\left\{A_{j}^{\prime}|| \mathcal{F}\left(A_{j}^{\prime}\right) \mid \geqslant 5\right\} \subseteq\left(\begin{array}{c}{[2 k-3]} \\ k-2\end{array}\right)$. Then it is easy to find two disjoint sets, one in $\mathcal{F}\left(A_{j_{1}}^{\prime}\right)$ and the other in $\mathcal{F}\left(A_{j_{2}}^{\prime}\right)$. This contradicts the assumption that $\mathcal{F}$ is intersecting. Therefore $|\mathcal{F}|=h(2 k+1,2 k-3, k)$.

Let us examine the maximum families. Note that $\alpha(m, 2 k-3, k)=h(m, 2 k-3, k)$ implies that inequality (1) becomes equality, $s=\left(\begin{array}{c}2 k-4 \\ k-2\end{array}\right)$, and $N-s=\left(\begin{array}{c}2 k-4 \\ k-3\end{array}\right)$. It follows 
that $\mathcal{F}\left(A_{j}^{\prime}\right)=\left\{A_{j}^{\prime} \cup B \mid B \in\left(\begin{array}{c}{[2 k-2, m]} \\ 2\end{array}\right)\right\}$ for $s<j \leqslant N$. Since there exists a nonintersecting pair $B_{1}$ and $B_{2}$ in $\left(\begin{array}{c}{[2 k-2, m]} \\ 2\end{array}\right),\left\{A_{j}^{\prime} \mid s+1 \leqslant j \leqslant N\right\}$ must be a maximum intersecting family in view of its cardinality. By Theorem 1 , there exists $t \in \cap_{j=s+1}^{N} A_{j}^{\prime}$. For $1 \leqslant j \leqslant s$, if there exists $\mathcal{F}\left(A_{j_{1}}^{\prime}\right) \neq \emptyset$ for some $1 \leqslant j_{1} \leqslant s$, then there exists some $A_{j_{2}}^{\prime}, s+1 \leqslant j_{2} \leqslant N$ such that $A_{j_{1}}^{\prime} \cap A_{j_{2}}^{\prime}=\emptyset$. We can find two disjoint sets, one in $\mathcal{F}\left(A_{j_{1}}^{\prime}\right)$ and the other in $\mathcal{F}\left(A_{j_{2}}^{\prime}\right)$, a contradiction. Therefore we have $\mathcal{F}\left(A_{j}^{\prime}\right)=\emptyset$ and $\mathcal{F}\left(A_{j}\right)=\left\{A_{j} \cup B \mid B \in\left(\begin{array}{c}{[2 k-2, m]} \\ 1\end{array}\right)\right\}$ for $1 \leqslant j \leqslant s$. Suppose that $t \notin A_{j_{0}}$ for some $1 \leqslant j_{0} \leqslant s$. Then $t \in A_{j_{0}}^{\prime}$. For any $A_{j}^{\prime}, s+1 \leqslant j \leqslant N$, we have $A_{j_{0}}^{\prime} \neq A_{j}^{\prime}$ since $\mathcal{F}\left(A_{j}\right)=\emptyset$, yet $\mathcal{F}\left(A_{j_{0}}\right) \neq \emptyset$. Then $\left\{A_{j_{0}}^{\prime}, A_{s+1}, \ldots, A_{N}\right\}$ is an intersecting family in $\left(\begin{array}{c}{[2 k-3]} \\ k-2\end{array}\right)$ having more than $\left(\begin{array}{c}2 k-4 \\ k-3\end{array}\right)$ members, a contradiction. Hence $\mathcal{F}$ has the form $\mathcal{H}_{t}$ for $t \in[2 k-3]$.

\section{The case for $m$ sufficiently large}

We have solved Problem 3 for $n=2 k-1,2 k-2$, and $2 k-3$. In this section, we are going to assume that $k \leqslant n<2 k-3$ and solve the problem when $m$ is sufficiently large.

Let $r, l, n$ be positive integers satisfying $r<l \leqslant n / 2$, and let $X_{1}$ and $X_{2}$ be disjoint $n$-sets. Wang and Zhang [11] characterized the maximum intersecting families $\mathcal{F} \subseteq\{F \in$ $\left(\begin{array}{c}X_{1} \cup X_{2} \\ r+l\end{array}\right)|| F \cap X_{1} \mid=r$ or $\left.l\right\}$ of maximum cardinality. We consider a similar extremal problem.

Problem 12. Given integers $m, n, k, c, d$ satisfying $n<m, k \leqslant n<2 k-3, d<c<k$, and $c+d=n$, characterize the intersecting families $\mathcal{F} \subseteq\left\{F \in\left(\begin{array}{c}{[m]} \\ k\end{array}\right)|| F \cap[n] \mid=c\right.$ or $\left.d\right\}$ of maximum cardinality.

We can derive an asymptotic solution of the above problem as follows.

Lemma 13. For given $n, k, c, d$ satisfying conditions in the above problem, if $m$ is sufficiently large, then a maximum intersecting family $\mathcal{F}$ has the form $\{A \cup B \cup\{t\} \mid A \in$ $\left.\left(\begin{array}{c}{[n] \backslash\{t\}} \\ c-1\end{array}\right), B \in\left(\begin{array}{c}{[n+1, m]} \\ k-c\end{array}\right)\right\} \cup\left\{A \cup B \cup\{t\} \mid A \in\left(\begin{array}{c}{[n] \backslash\{t\}} \\ d-1\end{array}\right), B \in\left(\begin{array}{c}{[n+1, m]} \\ k-d\end{array}\right)\right\}$ for some $t \in[n]$, and hence $|\mathcal{F}|=\left(\begin{array}{c}n-1 \\ c-1\end{array}\right)\left(\begin{array}{c}m-n \\ k-c\end{array}\right)+\left(\begin{array}{c}n-1 \\ d-1\end{array}\right)\left(\begin{array}{c}m-n \\ k-d\end{array}\right)$.

Proof. Let $\mathcal{F}$ be a maximum intersecting family satisfying the conditions of Problem 12 . Any special form stated in the lemma is an intersecting family, hence its cardinality $\left(\begin{array}{c}n-1 \\ c-1\end{array}\right)\left(\begin{array}{c}m-n \\ k-c\end{array}\right)+\left(\begin{array}{c}n-1 \\ d-1\end{array}\right)\left(\begin{array}{c}m-n \\ k-d\end{array}\right)$ supplies a lower bound for $|\mathcal{F}|$.

Let us consider upper bounds for $|\mathcal{F}|$. First partition $\mathcal{F}$ into two subfamilies $\mathcal{F}_{k-c}$ and $\mathcal{F}_{k-d}$ such that $\mathcal{F}_{k-c}=\{F \in \mathcal{F}|| F \cap[n] \mid=c\}$ and $\mathcal{F}_{k-d}=\{F \in \mathcal{F}|| F \cap[n] \mid=d\}$. For $\mathcal{F}_{k-d}$, we consider the injection from $\mathcal{F}_{k-d}$ to the vertex set of $\mathrm{KG}(n, d) \times \operatorname{KG}(m-n, k-d)$ defined prior to Lemma 6 . We may choose $m$ sufficiently large so that $2(k-d)<m-n$ and $m>n k / d$ hold. By Lemma 6 , we have $\left|\mathcal{F}_{k-d}\right| \leqslant \alpha(\operatorname{KG}(n, d))|\operatorname{KG}(m-n, k-d)|=$ $\left(\begin{array}{c}n-1 \\ d-1\end{array}\right)\left(\begin{array}{c}m-n \\ k-d\end{array}\right)$. Consider a further partition on $\mathcal{F}_{k-c}$ and $\mathcal{F}_{k-d}$. Denote $N=\left(\begin{array}{l}n \\ c\end{array}\right)$. For $A_{j} \in\left(\begin{array}{c}{[n]} \\ c\end{array}\right)$ and $A_{j}^{\prime}=[n] \backslash A_{j}, 1 \leqslant j \leqslant N$, let $\mathcal{F}\left(A_{j}\right)=\left\{F \in \mathcal{F}_{k-c} \mid F \cap[n]=A_{j}\right\}$ and $\mathcal{F}\left(A_{j}^{\prime}\right)=\left\{F \in \mathcal{F}_{k-d} \mid F \cap[n]=A_{j}^{\prime}\right\}$. Since $A_{j} \cap A_{j}^{\prime}=\emptyset$, the two families $\left\{B \in\left(\begin{array}{c}{[n+1, m]} \\ k-c\end{array}\right) \mid\right.$ $\left.A_{j} \cup B \in \mathcal{F}\right\}$ and $\left\{B \in\left(\begin{array}{c}{[n+1, m]} \\ k-d\end{array}\right) \mid A_{j}^{\prime} \cup B \in \mathcal{F}\right\}$ are cross-intersecting of size $\left|\mathcal{F}\left(A_{j}\right)\right|$ and 
$\left|\mathcal{F}\left(A_{j}^{\prime}\right)\right|$, respectively. Let $r \leqslant s$ be integers such that $\mathcal{F}\left(A_{j}\right)=\emptyset$ for $1 \leqslant j \leqslant r, \mathcal{F}\left(A_{j}\right)$ and $\mathcal{F}\left(A_{j}^{\prime}\right)$ are nonempty for $r+1 \leqslant j \leqslant s$ and $\mathcal{F}\left(A_{j}^{\prime}\right)=\emptyset$ for $s+1 \leqslant j \leqslant N$. Then by Theorem 7,

$$
\begin{aligned}
|\mathcal{F}|= & \sum_{j=1}^{r}\left|\mathcal{F}\left(A_{j}^{\prime}\right)\right|+\sum_{j=r+1}^{s}\left(\left|\mathcal{F}\left(A_{j}\right)\right|+\left|\mathcal{F}\left(A_{j}^{\prime}\right)\right|\right)+\sum_{j=s+1}^{N}\left|\mathcal{F}\left(A_{j}\right)\right| \\
\leqslant & r\left(\begin{array}{c}
m-n \\
k-d
\end{array}\right)+(s-r)\left(\left(\begin{array}{c}
m-n \\
k-d
\end{array}\right)-\left(\begin{array}{c}
m-k-d \\
k-d
\end{array}\right)+1\right) \\
& +(N-s)\left(\begin{array}{c}
m-n \\
k-c
\end{array}\right) .
\end{aligned}
$$

We first show that $r=\left(\begin{array}{l}n-1 \\ d-1\end{array}\right)$. If $r>\left(\begin{array}{c}n-1 \\ d-1\end{array}\right)$, then

$$
\begin{aligned}
|\mathcal{F}| & =\sum_{j=r+1}^{N}\left|\mathcal{F}\left(A_{j}\right)\right|+\sum_{j=1}^{s}\left|\mathcal{F}\left(A_{j}^{\prime}\right)\right| \\
& \leqslant(N-r)\left(\begin{array}{c}
m-n \\
k-c
\end{array}\right)+\left|\mathcal{F}_{k-d}\right| \\
& <\left(\begin{array}{c}
n-1 \\
c-1
\end{array}\right)\left(\begin{array}{c}
m-n \\
k-c
\end{array}\right)+\left(\begin{array}{c}
n-1 \\
d-1
\end{array}\right)\left(\begin{array}{c}
m-n \\
k-d
\end{array}\right),
\end{aligned}
$$

which cannot be true.

For $m$ sufficient large, say $m>2 n(n / 2)^{k-d}\left(\begin{array}{c}n \\ \lfloor n / 2\rfloor\end{array}\right)$, we have

$$
\begin{aligned}
& (s-r)\left(\left(\begin{array}{c}
m-n \\
k-d
\end{array}\right)-\left(\begin{array}{c}
m-k-d \\
k-d
\end{array}\right)+1\right)+(N-s)\left(\begin{array}{c}
m-n \\
k-c
\end{array}\right) \\
< & (s-r)\left(\frac{m^{k-d}}{(k-d) !}-\frac{(m-2 n)^{k-d}}{(k-d) !}+1\right)+(N-s) m^{k-c} \\
< & (s-r)\left(2 n m^{k-d-1}+1\right)+(N-s) m^{k-c} \\
< & N(2 n) m^{k-d-1} \\
\leqslant & \left(\begin{array}{c}
n \\
\lfloor n / 2\rfloor
\end{array}\right)(2 n)(n / 2)^{k-d} \frac{1}{m} \frac{m^{k-d}}{(n / 2)^{k-d}} \\
< & \left(\begin{array}{c}
m-n \\
k-d
\end{array}\right) .
\end{aligned}
$$

If $r<\left(\begin{array}{c}n-1 \\ d-1\end{array}\right)$, then $|\mathcal{F}|<(1+r)\left(\begin{array}{c}m-n \\ k-d\end{array}\right) \leqslant\left(\begin{array}{c}n-1 \\ d-1\end{array}\right)\left(\begin{array}{c}m-n \\ k-d\end{array}\right)$, which is impossible. Hence $r=\left(\begin{array}{c}n-1 \\ d-1\end{array}\right)$. Now we show that $s=\left(\begin{array}{l}n-1 \\ d-1\end{array}\right)$. Note that $s \geqslant r=\left(\begin{array}{l}n-1 \\ d-1\end{array}\right)$. Suppose $s>\left(\begin{array}{l}n-1 \\ d-1\end{array}\right)$. Then by Theorem 5 , the image of the injection from $\mathcal{F}_{k-d}$ to $\mathrm{KG}(n, d) \times \mathrm{KG}(m-n, k-d)$ cannot be a maximal independent set and $\left|\mathcal{F}_{k-d}\right|<\left(\begin{array}{c}n-1 \\ d-1\end{array}\right)\left(\begin{array}{c}m-n \\ k-d\end{array}\right)$. This leads to $|\mathcal{F}| \leqslant$ $(N-r)\left(\begin{array}{c}m-n \\ k-c\end{array}\right)+\left|\mathcal{F}_{k-d}\right|<\left(\begin{array}{c}n-1 \\ c-1\end{array}\right)\left(\begin{array}{c}m-n \\ k-c\end{array}\right)+\left(\begin{array}{c}n-1 \\ d-1\end{array}\right)\left(\begin{array}{c}m-n \\ k-d\end{array}\right)$, contradicting the lower bound of $|\mathcal{F}|$ again. Since $r=s=\left(\begin{array}{c}n-1 \\ d-1\end{array}\right)$, we have $|\mathcal{F}| \leqslant\left(\begin{array}{c}n-1 \\ d-1\end{array}\right)\left(\begin{array}{c}m-n \\ k-d\end{array}\right)+\left(\begin{array}{c}n-1 \\ c-1\end{array}\right)\left(\begin{array}{c}m-n \\ k-c\end{array}\right)$. The equality must hold as the right hand side is the known lower bound of $|\mathcal{F}|$. 
When $\mathcal{F}$ has maximum cardinality, $\mathcal{F}\left(A_{j}\right)=\left\{A_{j} \cup B \mid B \in\left(\begin{array}{c}{[n+1, m]} \\ k-c\end{array}\right)\right\}$ for $j>\left(\begin{array}{c}n-1 \\ d-1\end{array}\right)$ and $\mathcal{F}\left(A_{j}^{\prime}\right)=\left\{A_{j}^{\prime} \cup B \mid B \in\left(\begin{array}{c}{[n+1, m]} \\ k-d\end{array}\right)\right\}$ for $j \leqslant\left(\begin{array}{c}n-1 \\ d-1\end{array}\right)$. Now $\left\{A_{j}^{\prime} \mid 1 \leqslant j \leqslant\left(\begin{array}{c}n-1 \\ d-1\end{array}\right)\right\} \subseteq\left(\begin{array}{c}{[n]} \\ k\end{array}\right)$ is a maximum intersecting family. Thus, there is a common element $t \in A_{j}^{\prime}$ for $1 \leqslant j \leqslant\left(\begin{array}{l}n-1 \\ d-1\end{array}\right)$. On the other hand, no $A_{j}^{\prime}$ contains $t$ for $j>\left(\begin{array}{l}n-1 \\ d-1\end{array}\right)$. That implies $t \in A_{j}$. So $t$ belongs to every member of $\mathcal{F}$.

Theorem 14. If integers $n$ and $k$ satisfy $k \leqslant n<2 k-3$, then $\alpha(m, n, k)=h(m, n, k)$ holds for sufficiently large $m$. For such a large $m$, a maximum $(m, n, k)$-intersecting family is of the form $\mathcal{H}_{t}$ for some $t \in[n]$.

Proof. Let an $(m, n, k)$-intersecting family $\mathcal{F}$ have canonical partition $\left(\begin{array}{c}{[n]} \\ k\end{array}\right) \cup\left(\bigcup_{i=1}^{2 k-n-1} \mathcal{F}_{i}\right)$ as before. When $n$ is odd, we put $\mathcal{F}_{i}$ and $\mathcal{F}_{2 k-n-i}$ into a pair for $1 \leqslant i \leqslant(2 k-n-1) / 2$. When $n$ is even, we put $\mathcal{F}_{i}$ and $\mathcal{F}_{2 k-n-i}$ into a pair for $1 \leqslant i \leqslant\lfloor(2 k-n-1) / 2\rfloor-1$, and leave $\mathcal{F}_{\lfloor(2 k-n-1) / 2\rfloor}$ unpaired.

Let $c=k-i$ and $d=n-k+i$. The subfamily $\mathcal{F}_{i} \cup \mathcal{F}_{2 k-n-i}$ is an intersecting family and satisfies the conditions in Lemma 13. Therefore $\left|\mathcal{F}_{i}\right|+\left|\mathcal{F}_{2 k-n-i}\right| \leqslant\left(\begin{array}{c}n-1 \\ k-i-1\end{array}\right)\left(\begin{array}{c}m-n \\ i\end{array}\right)+$ $\left(\begin{array}{c}n-1 \\ n-k+i-1\end{array}\right)\left(\begin{array}{c}m-n \\ 2 k-n-i\end{array}\right)$ for sufficiently large $m$. When $n$ is odd, we immediately have the following.

$$
\begin{aligned}
|\mathcal{F}| & \leqslant\left(\begin{array}{l}
n \\
k
\end{array}\right)+\sum_{i=1}^{(2 k-n-1) / 2}\left(\begin{array}{c}
n-1 \\
k-i-1
\end{array}\right)\left(\begin{array}{c}
m-n \\
i
\end{array}\right)+\left(\begin{array}{c}
n-1 \\
k-i
\end{array}\right)\left(\begin{array}{c}
m-n \\
2 k-n-i
\end{array}\right) \\
& =\left(\begin{array}{l}
n \\
k
\end{array}\right)+\sum_{i=1}^{2 k-n-1}\left(\begin{array}{c}
n-1 \\
k-i-1
\end{array}\right)\left(\begin{array}{c}
m-n \\
i
\end{array}\right) .
\end{aligned}
$$

When $n$ is even, we have $\left|\mathcal{F}_{i}\right| \leqslant\left(\begin{array}{c}n-1 \\ k-i-1\end{array}\right)\left(\begin{array}{c}m-n \\ i\end{array}\right)$ for $i=\lfloor(2 k-n-1) / 2\rfloor$ by Theorem 5. Together with other upper bounds of $\left|\mathcal{F}_{i} \cup \mathcal{F}_{2 k-n-i}\right|$, we have shown $|\mathcal{F}| \leqslant\left(\begin{array}{l}n \\ k\end{array}\right)+$ $\sum_{i=1}^{2 k-n-1}\left(\begin{array}{c}n-1 \\ k-i-1\end{array}\right)\left(\begin{array}{c}m-n \\ i\end{array}\right)$.

When $\mathcal{F}$ is a maximum $(m, n, k)$-intersecting family, for each pair $\mathcal{F}_{i}$ and $\mathcal{F}_{2 k-n-i}$, there is an element $t_{i}$ belonging to every member of $\mathcal{F}_{i} \cup \mathcal{F}_{2 k-n-i}$. This also holds for $\mathcal{F}_{i}$, $i=\lfloor(2 k-n-1) / 2\rfloor$ for even $n$. Suppose that there exist $\mathcal{F}_{i_{1}} \cup \mathcal{F}_{2 k-n-i_{1}}$ and $\mathcal{F}_{i_{2}} \cup \mathcal{F}_{2 k-n-i_{2}}$ for which $t_{i_{1}} \neq t_{i_{2}}$. (The case that one of them is $\mathcal{F}_{i}, i=\lfloor(2 k-n-1) / 2\rfloor$ for even $n$, is the same.) Note that

$$
\mathcal{F}_{2 k-n-i_{j}}=\left\{A \cup B \cup\left\{t_{i_{j}}\right\} \mid A \in\left(\begin{array}{c}
{[n] \backslash\left\{t_{i_{j}}\right\}} \\
n-k+i_{j}-1
\end{array}\right), B \in\left(\begin{array}{c}
{[n+1, m]} \\
2 k-n-i_{j}
\end{array}\right)\right\}
$$

for $j=1,2$. Since $2\left(n-k+i_{j}-1\right) \leqslant n-1$ and $2\left(2 k-n-i_{j}\right)<m-n$, we can find subsets $F_{j} \in \mathcal{F}_{2 k-n-i_{j}}$ for $j=1,2$ such that $F_{1} \cap F_{2}=\emptyset$ if $t_{i_{1}} \neq t_{i_{2}}$. Therefore $t_{i_{1}} \neq t_{i_{2}}$ cannot happen. Consequently, $\mathcal{F}=\mathcal{H}_{t}$ for some $t \in[n]$.

\section{Conclusion}

We have introduced the notion of an $(m, n, k)$-intersecting family and studied its maximum cardinality $\alpha(m, n, k)$. The well-known theorems of Erdős-Ko-Rado and Hilton-Milner in 
finite extremal set theory are special cases for $n=k$ and $n=k+1$. The common cardinality $h(m, n, k)$ of a particular collection of $(m, n, k)$-intersecting families $\mathcal{H}_{t}^{m, n, k}$ supplies a natural lower bound for $\alpha(m, n, k)$. A noticeable feature of $\mathcal{H}_{t}^{m, n, k}$ is that members of $\mathcal{H}_{t}^{m, n, k} \backslash\left(\begin{array}{c}{[n]} \\ k\end{array}\right)$ have a nonempty intersection. We have proved that the families $\mathcal{H}_{t}^{m, n, k}$ are precisely all the $(m, n, k)$-intersecting families of maximum cardinality for the cases $n=2 k-1,2 k-3$, or $m$ sufficiently large. When $n=2 k-2$, there are other maximum families. Whether $\alpha(m, n, k)=h(m, n, k)$ is true in all cases and $\mathcal{H}_{t}^{m, n, k}, n \neq 2 k-2$, always characterizes maximum families are interesting open problems. Analogue problems can be formulated with respect to intersecting families having intersection size greater than some prescribed positive integer.

\section{Acknowledgements}

This work was done while the first author was a post-doctoral fellow in the Institute of Mathematics, Academia Sinica. The supports provided by the Institute is greatly appreciated.

\section{References}

[1] R. Ahlswede and L. H. Khachatrian. The complete nontrivial-intersection theorem for systems of finite sets. J. Combin. Theory Ser. A, 76:121-138, 1996.

[2] R. Ahlswede and L. H. Khachatrian. The complete intersection theorem for systems of finite sets. European J. Combin., 18:125-136, 1997.

[3] P. Borg. Intersecting families of sets and permutations: a survey, In A. R. Baswell (Ed.), Advances in Mathematics Research, volume 16, pages 283-299, Nova Science Publishers, 2011.

[4] M. Deza and P. Frankl. Erdős-Ko-Rado theorem - 22 years later. SIAM J. Algebraic Discrete Methods, 4:419-431, 1983.

[5] P. Erdős, C. Ko, and R. Rado. Intersection theorems for systems of finite sets. Quart. J. Math. Oxford Ser. 2, 12:313-320, 1961.

[6] P. Frankl. The Erdős-Ko-Rado Theorem is true for $n=$ ckt. Proc. Fifth Hung. Comb. Coll., pages 365-375, North-Holland, Amsterdam, 1978.

[7] P. Frankl. The shifting technique in extremal set theory. In C. Whitehead (Ed.), Combinatorial Surveys, pages 81-110, Cambridge Univ. Press, 1987.

[8] P. Frankl. An Erdős-Ko-Rado theorem for direct products. European J. Combin., 17:727-730, 1996.

[9] P. Frankl and N. Tokushige. Some best possible inequality concerning crossintersecting families. J. Combin. Theory Ser. A, 61:87-97, 1992.

[10] A. J. W. Hilton and E. C. Milner. Some intersection theorems for systems of finite sets. Quart. J. Math. Oxford Ser. 2, 18:369-384, 1967. 
[11] J. Wang and H. Zhang. Intersecting families in a subset of Boolean lattice. Electron. J. Combin., 18:\#P237, 2011.

[12] R. M. Wilson. The exact bound in the Erdős-Ko-Rado theorem. Combinatorica, 4:247-257, 1984.

[13] H. Zhang. Independent sets in direct products of vertex-transitive graphs. J. Combin. Theory Ser. B, 102:832-838, 2012. 